\title{
Research on the Technology of Modern City Subway Inquiry System Based on Java Programming
}

\author{
Wanzhen Zhang ${ }^{1, \text { a }}$, Yufei Wang ${ }^{1, b}$ and Kun Zhang ${ }^{1 c^{*}}$ \\ ${ }^{1}$ College of Computer Engineering, Hainan Tropical Ocean University, Sanya, Hainan, 572022, China \\ a107689990@qq.com, b1576949369@qq.com, 'zk0588@163.com \\ ${ }^{*}$ The corresponding author
}

Keywords: Rail transit; Intelligent data management; Program design; Urban transport; Query system.

\begin{abstract}
With the development of China's transportation industry, the formation of a number of scale of the road to the city subway enterprises, they continue to pursue a higher level. For modern urban subway route planner and ordinary users to use the urban subway query system design and application, software design provides including subway lines management and maintenance, MTR Corporation Information Management and maintenance, subway information management and maintenance, while providing all types of queries, and query results in preferential ordering principle of intelligent query, can basically meet the needs of urban subway line inquiry system function is required.
\end{abstract}

\section{Introduction}

City subway is a special service for the public transport enterprises, with the rapid development of the national economy and urban construction, urban economic prosperity, population increase, the city must solve the needs of people travel. Urban metro is directly related to the city's economic development and people's life, has a global influence and guiding of urban economy, urban subway with its convenient, fast and characteristics of large capacity and become the theme of urban traffic. But with the huge subway system, it is very difficult to get accurate information on the subway, so that some people travel to bring inconvenience. Therefore, in urgent need of a convenient and fast way of city subway information query, the system can achieve the basic functions of the subway inquiry. Reducing travel time will make all the subway users produce benefits, fast traffic, better information and better market can improve the image of the city subway, can increase the subway ride. Urban subway transportation with its wide coverage characteristics, become the first choice for the vast majority of travelers. Especially foreign tourism, travel, medical care, need to understand the local road conditions can use this system is convenient query all meet their requirements of subway, to provide assistance for their travel and life.

Query the metro system development is in a backward level, the majority of the passengers can get the subway, subway information completeness and accuracy cannot be guaranteed. Moreover, there is no specialized agencies responsible for the dissemination of information and management. However, it is also a big problem for the city that he is not familiar with, so it is very important to develop a city subway system. Therefore, our team chose to Java technology to achieve urban subway query system, but also to take this research and design of the University as a comprehensive application of knowledge. The core of the system is to carry out the route of the query, and the input to the station to inquire, click the "query" button, you can check all the stations have the subway line.

\section{Development Status at Home and Abroad}

At present, the domestic subway industry is facing rare opportunities for development. Countries for stimulating domestic demand, and vigorously promote the construction of public infrastructure, the construction of the subway also have been accelerated, after Beijing, Shanghai, Guangzhou, Shenzhen, 
Nanjing, Hangzhou, Chengdu, Shenyang, Nanning city began to subway construction, and soon there will be more metro put in operation. On the one hand, rail transport as a public service enterprises, and constantly improve the operational level and quality of service to become the urgent task of the subway. On the other hand, different types of customers will bring different service requirements. In this case, the demand for passengers not only put forward higher requirements to the subway service, but also trigger the business opportunities in the subway e-commerce applications. Therefore, from the improvement of the existing business operation system and means of early planning to establish a include passenger service, internal service management, electronic commerce and business management system application integrated passenger service information system in metro industry demand becomes more and more urgent.

This paper will focus on urban subway query system software design is described, which resources usury and system integrated and accurate service and for the future electronic commerce layout of Metro membership management is software implementation of key and difficult. Therefore, this paper will also several software design difficulties are analyzed and processed, and of the system implementation and testing, including the Java query module design and implementation, and database design and Optimization Based on.

\section{Technology Introduction and System Analysis}

Java language is a popular programming language, it has the characteristics of object-oriented, cross platform, distributed application, and so on. The emergence of Java led to a revolution in the field of programming, which was hailed as one of the most important technologies in the twentieth Century. Java can not only small application program is compiled to realize sound and animation capabilities of embedded in web pages, but also can be used to separate large and medium application program and its powerful function of network to the entire Internet as a unified operation platform, greatly expanded the connotation and extension of traditional stand-alone or mode of client / server applications. Since the 1995 officially published, Java has gradually from a simple high-level computer programming language development for an important platform for the operation, and thus trigger and Java industry led to the development and growth, become a force that cannot be ignored in today's computer industry and important hair development trend and direction.

At the beginning of the system design, we must first consider how to construct the structure and semantics of the database with the data model. The widely used data model can be divided into two categories: a class is independent of the computer system of "conceptual data model, such as the entity relationship model, and another is directly facing the logical structure of the database structure data model". However, in this system, I used the "entity relation model" to describe the structure and semantics of the database, and the data abstraction. Entity relation model directly from the real world abstract entity type and entity relation, then use E-R diagram to show the model of the data. It has two obvious advantages: easy to understand and easy to accept. However, the entity relation model can only explain the relationship between entities, which cannot explain the internal data structure in detail. It is only the first step in the design of database. Through needs analysis can be learned from the user to the basic information content and requirements of the project, which is the premise of the follow-up work of the project. Demand analysis is very important in the design procedure, we based on Java in the subway query system of demand analysis. Analysis its feasibility and importance, and the system of each module to complete the analysis and design.

The system has two roles: the general user and administrator. Users by account and password will be able to carry out the subway information query, such as: subway line information (including the basic information and the line information query), query after a site every subway information, transfer scheme query, view the Metro news. Administrators not only have the general user's query function, but also has the function of line management and maintenance, such as: line site management, line attribute information management, news release, etc. The city subway inquiry system is mainly three functions, respectively: line management, site management, message management. Line management: 
users can find the site through the site, but also can directly view the site owned by the site. Administrators can add, delete and modify the line. Site management: the user can through the site to find the site, arrange travel route. Administrators can add, delete and modify the site. News release: the administrator can update the system message through the news release function.

Under the premise of the system target and database structure, the main functions of the system are designed: system login, data entry and modification, data comprehensive query, data statistics, etc.. To $\log$ on to the system and management, for example, in order to ensure the confidentiality and security of user data to prevent illegal users to the malicious data destruction, the system will be used to $\log$ in to the system, the designed well in advance of the user name and password information placed in the data table. The system automatically compares the information of the user input with the data in the data table, only the legitimate user can log in to the system and use the system. Through the system management users can achieve user login password changes and other functions. Data input, delete and modify data collectively referred to as management, the user can according to their own needs, the table data in the data management, will change the data in a timely manner to spread to the background database, to ensure that the data real-time, effective. Query function is a core part of the system, reflecting the good or bad of a system. This system can inquire about the subway line information, the subway line information, the transfer information and the site.

\section{Database Design}

In view of the widespread application and scalability, system uses the open source SQL database management system, can not only meet the largest data processing system and commercial web site data storage needs, but also for individuals or small businesses provide easy-to-use data storage services. The system is mainly to create 5 tables: the subway table, station info table, s_user table, the subway line table and transfer table.

Subway Table. The main station of the subway station, terminal station, terminal station, subway line number, ticket price and operation time, when the user inquires the subway line information needs to visit this table, as shown in Table 1.

Table 1 Subway table

\begin{tabular}{lcccc}
\hline Field name & Data type & Length & Space & Key words \\
\hline Subway line number & int & 4 & N & Y \\
Departure Station & varchar & 50 & N & Y \\
Terminus & varchar & 50 & N & Y \\
Ticket Price & varchar & 50 & N & Y \\
Ticket Price & varchar & 50 & N & Y \\
\hline
\end{tabular}

Transfer Table. Transfer table is mainly stored in the first number, transfer station, transfer to the subway station and the number of terminal. When the user inquires the line, the realization of the 1 transfer query function to access the table, as shown in Table 2. 
Table 2 Transfer table

\begin{tabular}{lcccc}
\hline Field name & Data type & Length & Space & Key words \\
\hline First by line number & int & 4 & $\mathrm{~N}$ & $\mathrm{Y}$ \\
Transfer station & varchar & 50 & $\mathrm{~N}$ & $\mathrm{~N}$ \\
Transfer metro & int & 4 & $\mathrm{~N}$ & $\mathrm{~N}$ \\
Terminal number & int & 50 & $\mathrm{~N}$ & $\mathrm{~N}$ \\
\hline
\end{tabular}

Stationinfo Table. Stationinfo table main storage site and subway lines have the site, for the user to check the site and the subway line access to this table, as shown in Table 3.

Table 3 Stationinfo table

\begin{tabular}{lcccc}
\hline Field name & Data type & Length & Space & Key words \\
\hline Subway line number & int & 4 & $\mathrm{~N}$ & $\mathrm{Y}$ \\
Subway line number & varchar & 50 & $\mathrm{~N}$ & $\mathrm{~N}$ \\
The name of the station & int & 4 & $\mathrm{~N}$ & $\mathrm{~N}$ \\
\hline
\end{tabular}

S_ user Table. $S_{-}$user table is required to store the user name and password login system, the need to access the background when the table, as shown in Table 4.

Table 4 S_ user table

\begin{tabular}{lcccc}
\hline Field name & Data type & Length & Space & Key words \\
\hline Password & varchar & 50 & $\mathrm{~N}$ & $\mathrm{~N}$ \\
Username & varchar & 50 & $\mathrm{~N}$ & $\mathrm{~N}$ \\
\hline
\end{tabular}

Subway Line Table. Subway line number and serial number, the user inquires the subway line to visit this table, as shown in Table 5.

Table 5 Subway line table

\begin{tabular}{lcccc}
\hline Field name & Data type & Length & Space & Key words \\
\hline Subway line number & int & 4 & N & Y \\
Serial number & varchar & 50 & N & N \\
\hline
\end{tabular}

\section{System Module Function Overview}

We designed the city subway system, including the system login module, password modification module, the subway line query module, the subway station query module and add the line, modify the line, delete the line. After login successfully, enter the system main interface, the user can carry on the site inquiry, the line inquiry and the drive inquiry, and can view the help, the subway consultation and the Metro Inc information. Subway line information maintenance module consists of the following parts: the subway information view and the subway line information modification function, its main function is to maintain the operation of the subway line information. Metro Line Information View function: click on the left menu tree of the subway information button that can view the subway line information. 


\section{Summary}

The era of big data, intelligent application software development news quickly, this paper as the new era college students majoring in computer science, innovation and entrepreneurship training college project team of a preliminary phase study results, in system of perfect and comprehensive application, run the mature of remains to be further improvement, in the future study and research process will be pay more attention to intelligent and practical combination.

\section{Acknowledgements}

The work was supported by the 2014 innovation and entrepreneurship training college national college projects (No. 201411100069).

\section{References}

[1] Wanzhen Zhang, Cui Wu, Jiayu Xiao, Query System Based on Modern Urban Subway Java Program Design, Software, Vol.37, No.3, pp. 40-43, 2016.

[2] Yunfeng Wang, Jing Wang, Shiwei Huang, Hardware implementation of a point multiplication algorithm based on parallel scheduling, The Journal of New Industrialization, Vol.4, No.7, pp. 20-27, 2014.

[3] Xiaojun Chen, Peide Lou, The Current Situation and Development Trend of In-vehicle, Software, Vol.35, No.10, pp. 95-99, 2014.

[4] Xingcheng Cui, Tong Zhao, Java Dynamic Class Loading Mechanism Analysis and Its Application, Computer Systems \& Applications, Vol.22 No.7, pp. 187-191, 2013.

[5] Hongjie Tang, The Research on Java Basic Grammar Teaching of Java Programming Language, Software, Vol.35, No.6, pp. 23-25, 2014.

[6] Huiling Han, to achieve a two-way parallel scheduling algorithm by hardware, North University of China, 2012.

[7] Peifeng Tao, Design and implementation of urban public traffic inquiry system, Central South University, 2008.

[8] Kun Zhang, Haifeng Wang, Shengpei Dai, Research on Tropical Agricultural Products Quality Recognition and Detection System based on Electronic Nose Technology, Advanced Materials Research. Vol. 659, pp. 75-78, 2013.

[9] Kun Zhang, Hongxu Wang, Haifeng Wang, Zhuang Li, The Time Series Prediction Algorithm of Enrollment based on the Hesitancy degree Correction Rule of Vague Sets, ICIC Express Letters, vol. 9, No. 5, pp. 1311-1318, 2015.

[10] Kun Zhang, Zhuang Li, Haifeng Wang, HongXu Wang, Fuzzy Time Series Prediction Model and Application based on Fuzzy Inverse, International Journal of Signal Processing, Image Processing and Pattern Recognition, vol. 8, No.10, pp. 121-128, 2015.

[11] Hui Mo, Design and development of public transport query system, Western China Communication Science \& Technology, No. 9, pp. 67-72, 2012. 